\title{
Dynamic Simulation of Human Thermoregulation and Heat Transfer for
}

\section{Spaceflight Applications}

\author{
Thomas R. Miller ${ }^{1}$, David A. Nelson ${ }^{1}$, Grant Bue ${ }^{2}$, Lawrence Kuznetz ${ }^{3}$ \\ ${ }^{1}$ Mechanical Engineering Department, University of South Alabama, Mobile, AL 36688-0002 \\ ${ }^{2}$ NASA Johnson Space Center, Houston, TX \\ ${ }^{3}$ Retired
}

Models of human thermoregulation and heat transfer date from the early 1970s and have been developed for applications ranging from evaluating thermal comfort in spacecraft and aircraft cabin environments to predicting heat stress during EVAs. Most lumped or compartment models represent the body as an assemblage cylindrical and spherical elements which may be subdivided into layers to describe tissue heterogeneity. Many existing models are of limited usefulness in asymmetric thermal environments, such as may be encountered during an EVA. Conventional whole-body clothing models also limit the ability to describe local surface thermal and evaporation effects in sufficient detail. A further limitation is that models based on a standard man model are not readily scalable to represent large or small subjects.

This work describes development of a new human thermal model derived from the 41node man model. Each segment is divided into four concentric, constant thickness cylinders made up of a central core surrounded by muscle, fat, and skin, respectively. These cylinders are connected by the flow of blood from a central blood pool to each part. The central blood pool is updated at each time step, based on a whole-body energy balance.

Results show the model simulates core and surface temperature histories, sweat evaporation and metabolic rates which generally are consistent with controlled exposures of human subjects. Scaling rules are developed to enable simulation of small and large subjects $\left(5^{\text {th }}\right.$ per centile and $95^{\text {th }}$ per centile). Future refinements will include a clothing model that addresses local surface insulation and permeation effects and developing control equations to describe thermoregulatory effects such as may occur with prolonged weightlessness or with aging. 


\title{
Dynamic Simulation of Human Thermoregulation and Heat Transfer for Spaceflight Applications
}

\author{
Thomas R. Miller ${ }^{1}$ and David A. Nelson ${ }^{2}$ \\ University of South Alabama, Mobile, Alabama, 36688 \\ Lawrence Kuznetz ${ }^{3}$ \\ Business or Academic Affiliation 2, City, Province, Zip Code, Country \\ and \\ Grant Bue ${ }^{4}$ \\ Business or Academic Affiliation 2, City, State, Zip Code
}

\begin{abstract}
Models of human thermoregulation and heat transfer date from the early 1970s and have been developed for applications ranging from evaluating thermal comfort in spacecraft and aircraft cabin environments to predicting heat stress during EVAs. Most lumped or compartment models represent the body as an assemblage cylindrical and spherical elements which may be subdivided into layers to describe tissue heterogeneity. Many existing models are of limited usefulness in asymmetric thermal environments, such as may be encountered during an EVA. Conventional whole-body clothing models also limit the ability to describe local surface thermal and evaporation effects in sufficient detail. A further limitation is that models based on a standard man model are not readily scalable to represent large or small subjects. This work describes development of a new human thermal model derived from the 41-node man model. Each segment is divided into four concentric, constant thickness cylinders made up of a central core surrounded by muscle, fat, and skin, respectively. These cylinders are connected by the flow of blood from a central blood pool to each part. The central blood pool is updated at each time step, based on a whole-body energy balance. Results show the model simulates core and surface temperature histories, sweat evaporation and metabolic rates which generally are consistent with controlled exposures of human subjects. Scaling rules are developed to enable simulation of small and large subjects $\left(5^{\text {th }}\right.$ per centile and $95^{\text {th }}$ per centile). Future refinements will include improvements to the clothing model that addresses local surface insulation and permeation effects and developing control equations to describe thermoregulatory effects such as may occur with prolonged weightlessness or with aging.
\end{abstract}

\section{Nomenclature}

$\begin{array}{ll}A T & =\text { Adipose tissue } \\ H T & =\text { Height of the subject } \\ L_{B P} & =\text { Length of a body segment } \\ L_{C, B P} & =\text { Length coefficient of a body segment } \\ N A T & =\text { Non-adipose tissue } \\ P B F & =\text { Percent body fat } \\ W T & =\text { Weight }\end{array}$

1 Research Assistant, Mechanical Engineering, 307 University Boulevard,Mobile, Alabama 36688-0002 (251) 460-6101, AIAA Member Grade for first author.

${ }^{2}$ Insert Job Title, Department Name, Address/Mail Stop, and AIAA Member Grade for second author.

${ }^{3}$ Insert Job Title, Department Name, Address/Mail Stop, and AIAA Member Grade for third author.

${ }^{4}$ Insert Job Title, Department Name, Address/Mail Stop, and AIAA Member Grade for fourth author (etc). 


\section{Introduction}

The National Aeronautics and Space Administration, NASA, is developing a new space suit to improve the suit's comfort and functionality during extended extravehicular activities, EVA's. ${ }^{1}$ EVA's can create unusual environmental conditions such as incident radiation shining upon one side of the astronaut with the other side facing deep space. An accurate mathematical model of the thermoregulatory state of an astronaut is needed to evaluate the performance of a new suit for the extreme and highly asymmetric environment the can occur during an EVA.

The current human thermal model used by NASA is the 41 Node Man, ${ }^{2}$ which is based on the physiology of the Stolwijk Model. ${ }^{3}$ The 41 Node Man added the capability of modeling a liquid cooled garment, a space suit, and variable anthropometrics. In the Stolwijk model, the human body is modeled as 10 segments. The head is modeled as a sphere, and each other body segment is modeled as a cylinder. There is a cylindrical segment for the torso and each arm, leg, hand, and foot. Each segment is further divided into 4 concentric, constant thickness cylinders made up of a central core surrounded by muscle, fat, and skin, respectively. These cylinders are connected by the flow of blood from a central blood pool to each part. There is no direct conduction between the separate body segments in the model. This is called a compartment model. The heat transfer rates between the concentric cylinders are calculated via conduction. The transient behavior is modeled using a lumped capacity method. The thermoregulation is modeled using control equations, which regulate the metabolic rates, blow flow rates, and sweating in an attempt to maintain homeostasis.

The 41 Node Man does not have the ability to account for the asymmetric environments that can be found in space, such as when an astronaut has radiation shining on one side of him and not the other. The Kuznetz twodimensional version of the 41 node $\operatorname{man}^{4}$ is an improvement because it does allow for asymmetric boundary conditions around the person by using a finite difference method to solve the heat transfer equations. In order to improve the temperature resolution and the results, Wissler ${ }^{5}$ developed a model that divided the human body into 15 cylindrical elements. Unlike the previous mentioned models, the Wissler model takes into account counter current heat exchange between major veins and arteries. Wissler split the torso into an upper and lower torso. The arms and legs were split into upper and lower pieces. The head, hands, and feet continued to be separate as in the Stolwijk Model. This model was published originally as a one-dimensional model and was later expanded to a twodimensional model ${ }^{6}$ that included angular variation in the cylinders similar to Kuznetz's two-dimensional model.

Fiala et al. developed an improvement to both the passive ${ }^{7}$ and active ${ }^{8}$ systems. The passive system is comprised of the tissues, blood flow, and metabolic rates of the body. The active system is a controller that regulates the blood flow and metabolic rates of the passive system. This passive model is one-dimensional but is separated into different sectors to allow for asymmetric boundary conditions. The body is divided into 15 cylindrical and spherical elements. These are the head, face, neck, shoulders, arms, hands, thorax, abdomen, legs, and feet ${ }^{7}$. The different body parts are composed of individual materials consisting of brain, lung, bone, muscle, viscera, fat, and skin. The active system controller was designed based on regression analysis from observed experimental data that measured the thermal responses of people to widely varying environmental conditions ${ }^{8}$. There have been some higher resolution threedimensional models such as the Visible Human Voxel Model ${ }^{9}$ and a model by Werner et al. ${ }^{10}$. The Voxel Model is anatomically correct and divides the body into $1.3 \times 10^{8}$ cubic volume elements (voxels) measuring $0.2 \mathrm{~cm}$ per side? The Werner model divides the body into a three dimensional grid with the nodes separated by $.5-1[\mathrm{~cm}]$.

Most of the prior models were designed to model a typical man approximately $74[\mathrm{~kg}]$ in weight, a surface area of $1.9\left[\mathrm{~m}^{2}\right]$, and a body fat of $14 \%$. Only the 41 Node Man is capable of simulating people of different anthropometrics. However, the 41 Node Man is not capable of modeling individuals in asymmetric environments such as those that can be encountered during an EVA. One objective of this research is to develop a model capable of simulating a range of individuals of different sizes in asymmetric environments.

Another requirement set by NASA is to develop the model using a new modern programming language. The 41 Node Man was created in FORTRAN, which is no longer widely supported. There are only a few people capable of using the 41 Node Man or modifying the source code ${ }^{1}$. The model must also be capable of being used by anyone with a little knowledge and expertise. This may require the use of a Graphical User Interface. The model itself needs to be able to run on a notebook or desktop computer. This means that large, high resolution models such as the Voxel Model are not practical. The large three dimensional models seem to be more for the purposes of studying localized internal temperature than predicting the overall thermal state of the subject. They use the same basic thermoregulatory system as the compartment models and apply it to a larger, more detailed physical model. A compartment model which idealizes the body as separate geometric shapes should provide enough resolution to meet the current requirements while keeping the computing requirements low enough to run on a personal computer. 


\section{Methods}

\section{A. General Description}

The model developed in this paper is a compartment model in which the human body is represented as the combination of a passive system and an active system, which controls the passive system. The foundation of the model was originally developed by Fiala et al. ${ }^{7,8}$. This present model extends the Fiala model by adding upper and lower limbs, angular heat transfer, and individualization in relation to height, weight, body fat percentage. Angular heat transfer is important for certain extreme environments, which can produce angular temperature gradients. ${ }^{4}$ The passive system in the current model consists of 19 separate body segments, and is shown schematically in Figure 1. Each body segment is further divided into concentric layers and angular sectors. The layers represent major tissue divides with depth, and the sectors allow the modeling of asymmetric boundary conditions .

\section{B. Individualization}

The Fiala "average man" is a person with a surface area of $1.86[\mathrm{~m} 2]$, a weight of $73.5[\mathrm{~kg}]$, and a body fat percentage of $14 \%$. The present model was individualized according to anthropometrics using a method by Montgomery 11 and also implemented in the 41 Node Man Model by Kuznetz 2. The user input is the percent body fat ,PBF; height ,HT [cm]; and weight, WT $[\mathrm{g}]$, of the subjects. The adipose tissue mass, AT $[\mathrm{kg}]$, and non-adipose tissue mass, NAT $[\mathrm{kg}]$, are calculated in equations 1 and 2 .

$$
\begin{gathered}
A T=(P B F)(W T)(.001) \\
N A T=(W T-(P B F)(W T))(.001)
\end{gathered}
$$

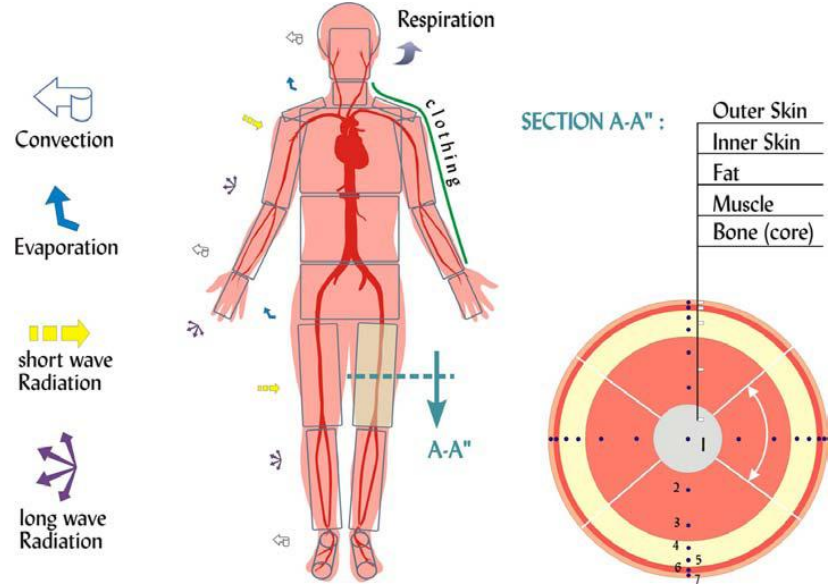

Figure 1. This figure shows the basic layout and construction of the passive system. The figure has been adapted from Fiala.
The relative weights of each compartment to the whole were distributed using the coefficients shown in Table 1 from the bodysize function of the 41 node man. Since in the current model, the head segment shown in Table 1 is split into the head, face, and neck. The relative distribution of tissues by weight from Fiala ${ }^{7}$ is used to divide the overall head segment coefficient into the different pieces. A similar approach was used for all the other segments as well. The resulting relative weight coefficient for all the segments is shown in Table 2.
Relative Weights of Each Body Compartment in the 41 Node Man

\begin{tabular}{|l|l|l|l|l|l|}
\hline & skeleton & viscera & muscle & fat & skin \\
\hline head & 0.019292 & 0.028232 & 0.00588 & 0.0333 & 0.00423 \\
\hline trunk & 0.04704 & 0.18704 & 0.2834 & 0.6333 & 0.0213 \\
\hline arm & 0.011878 & 0.005882 & 0.02664 & 0.04335 & 0.00382 \\
\hline arm & 0.011878 & 0.005882 & 0.02664 & 0.04335 & 0.00382 \\
\hline hand & 0.001824 & 0.000235 & 0.000594 & 0.006665 & 0.00147 \\
\hline hand & 0.001824 & 0.000235 & 0.000594 & 0.006665 & 0.00147 \\
\hline leg & 0.039584 & 0.015176 & 0.0805 & 0.10665 & 0.00947 \\
\hline leg & 0.039584 & 0.015176 & 0.0805 & 0.10665 & 0.00947 \\
\hline foot & 0.002942 & 0.000471 & 0.000594 & 0.01 & 0.00188 \\
\hline foot & 0.002942 & 0.000471 & 0.000594 & 0.01 & 0.00188 \\
\hline
\end{tabular}

Table 1. This table shows the relative weights of each compartment for individual segments of the body from Montgomery ${ }^{11}$ and Kuznetz ${ }^{2}$. 
Relative Weights of Each Body Compartment in the Present Model

\begin{tabular}{|c|c|c|c|c|c|c|}
\hline & muscle & skeleton & viscera & muscle & fat & skin \\
\hline head & 0 & 0.011960337 & 0.028232 & 0 & 0.009647 & 0.002546 \\
\hline face & 0.00067592 & 0.006030051 & 0 & 0.001587 & 0.020742 & 0.00106 \\
\hline neck & 0 & 0.001301612 & 0 & 0.003617 & 0.002911 & 0.000624 \\
\hline r shoulder & 0 & 0.002812211 & 0 & 0.000501 & 0.006624 & 0.000535 \\
\hline 1 shoulder & 0 & 0.002812211 & 0 & 0.000501 & 0.006624 & 0.000535 \\
\hline thorax & 0 & 0.021361923 & 0.042679 & 0.127263 & 0.071914 & 0.008856 \\
\hline abdomen & 0 & 0.015569175 & 0.144361 & 0.155135 & 0.548137 & 0.011374 \\
\hline upper arm & 0 & 0.006654542 & 0.003295 & 0.014925 & 0.024286 & 0.00214 \\
\hline lower arm & 0 & 0.005223458 & 0.002587 & 0.011715 & 0.019064 & 0.00168 \\
\hline upper arm & 0 & 0.006654542 & 0.003295 & 0.014925 & 0.024286 & 0.00214 \\
\hline lower arm & 0 & 0.005223458 & 0.002587 & 0.011715 & 0.019064 & 0.00168 \\
\hline hand & 0 & 0.001824 & 0.000235 & 0.000594 & 0.006665 & 0.00147 \\
\hline hand & 0 & 0.001824 & 0.000235 & 0.000594 & 0.006665 & 0.00147 \\
\hline upper leg & 0 & 0.01975169 & 0.007573 & 0.040168 & 0.053216 & 0.004725 \\
\hline lower leg & 0 & 0.01983231 & 0.007603 & 0.040332 & 0.053434 & 0.004745 \\
\hline upper leg & 0 & 0.01975169 & 0.007573 & 0.040168 & 0.053216 & 0.004725 \\
\hline lower leg & 0 & 0.01983231 & 0.007603 & 0.040332 & 0.053434 & 0.004745 \\
\hline foot & 0 & 0.002942 & 0.000471 & 0.000594 & 0.01 & 0.00188 \\
\hline foot & 0 & 0.002942 & 0.000471 & 0.000594 & 0.01 & 0.00188 \\
\hline
\end{tabular}

Table 2. This table shows the relative weight distribution coefficient for all the segments in the current model. It is understood that the precision of the numbers above is higher than physical accuracy allows. What is shown is mathematical results that occur because the coefficients must sum to 1.

To obtain the actual weight of each compartment, the fat coefficients are multiplied by $A T$, and all the other coefficients are multiplied by NAT. The volume of each compartment can then be obtained by dividing each compartment by the appropriate density for that material. The length of each cylindrical compartment, $L_{B P}$, is determined as a function of the individual's height according to Winter ${ }^{12}$.

$$
L_{B P}=L_{C, B P} H T
$$

Table 3 shows the length coefficients, $L_{c, B P}$, for each segment.

\section{Length Coefficients}

\begin{tabular}{|l|l|l|l|l|l|l|l|l|l|l|}
\hline face & neck & shoulder & thorax & abdomen & $\begin{array}{l}\text { upper } \\
\text { arm }\end{array}$ & $\begin{array}{l}\text { lower } \\
\text { arm }\end{array}$ & hand & $\begin{array}{l}\text { upper } \\
\text { leg }\end{array}$ & $\begin{array}{l}\text { lower } \\
\text { leg }\end{array}$ & foot \\
\hline 0.066 & 0.052 & 0.129 & 0.139 & 0.251 & 0.186 & 0.146 & 0.108 & 0.245 & 0.246 & 0.152 \\
\hline
\end{tabular}

Table 3. This table gives the length coefficients for the different body segments from Winter ${ }^{12}$.

With the volume and length of each segment known the outer radius, $r_{o}$, of each compartment can be calculated from the following geometric equations. 
This section has detailed the modelling of the human thermoregulatory system. The model is a compartment model consisting of 636 nodes. The spacial derivatives of the bio-heat transfer equation are approximated by a finite volume approach. The time derivative is approximated by a Crank-Nicolson approach. The model is capable of simulating asymetric boundary conditions, individuals, and different clothing ensembles.

With the volume and length of each segment known the outer radius, $r_{o}$, of each compartment can be calculated from simple geometry.

\section{Results}

The calculations were performed using a laptop PC. The code is written in Mathworks MatLab ${ }^{\text {TM }}$ version 2009. A number of experiments were simulated using different time steps and any time step between 0 and $20 \mathrm{~s}$ yielded results similar to each other. The model is unstable for any timestep larger due to the explicit blood pool calculation. Since the minimum timespan for the experimental results simulated in this report is 210 min., the largest possible timestep of 20 seconds is used in all the cases simulated in this paper. This timestep will provide enough resolution while minimizing the computing time.

\section{A. Thermoneutral Case}

Thermoneutral conditions for an average reclining and resting human in a typical indoor environment are defined by Fiala ${ }^{7}$. These conditions are an air and wall temperature of 30 [C], $40 \%$ relative humidity, an air velocity of $.05 \mathrm{~m} / \mathrm{s}$, and a whole body

Thermoneutral Results
\begin{tabular}{|l|l|l|l|l|l|}
\hline $\mathrm{M}$ & $\mathrm{T}_{\mathrm{hy}}$ & $T_{\text {skm.A }}$ & $T_{\text {skm.w }}$ & $\mathrm{T}_{\mathrm{rec}}$ & $\mathrm{T}_{\text {bl, } \mathrm{p}}$ \\
\hline 87.14 & 37.18 & 34.48 & 34.57 & 37.09 & 36.84 \\
\hline
\end{tabular}

Table 4. The table above shows the passive system steady state results in a thermoneutral environment.

metabolic rate of .8 met. The passive system was simulated in this environment from a constant temperature of 37 [C] to steady state to determine the set points used in the active system. The results of this simulation are shown in Table 4. The center node of the brain is considered to be the hypothalamus temperature, $T_{h y}$, and the center node of the abdomen is considered to be the rectal temperature, $T_{\text {rec }}$.

\section{B. Warm and Hot Cases}

Experimental tests performed by Stolwijk and Hardy ${ }^{13}$ were simulated to test the response of the model's active system in moderate to hot conditions. The physical model simulated in this task is the Fiala "average man" 7 . This should yield valid results because Stolwijk and Hardy present the average results from three subjects, and they note there is not a significant different in the recorded data between the subjects. The four experiments consisted of placing a subject wearing only shorts in an environment that is approximately $28[\mathrm{C}]$ for 1 [hr], then transferring them to a higher temperature environment of approximately 33, 38, 43, and 48 [C] environments for 2 [hr], then back to the $28[\mathrm{C}]$ environment for 1 [hr]. See Table 5 for more details. The starting conditions for the simulation were thermoneutral as detailed in Fiala ${ }^{7}$ Error! Reference source not found.. Stolwijk and Hardy recorded values for the metabolic rate of approximately .8 met. However, as noted by Nelson et al. ${ }^{9}$ and Fiala ${ }^{8}$, the typical metabolic rate for an awake, seated person is 1 met. The results for both metabolic rates for mean skin temperature and rectal temperature comparing the measured and simulated values are shown in Figures 2 and 3.

Stolwijk and Hardy Exposure Conditions

\begin{tabular}{|l|l|l|l|}
\hline & Pre-exposure & Exposure & Post Exposure \\
\hline Warm (W) & $27.8^{\circ} \mathrm{C}$ at $37 \% \mathrm{RH}$ & $33.3{ }^{\circ} \mathrm{C}$ at $34 \% \mathrm{RH}$ & $28.0{ }^{\circ} \mathrm{C}$ at $37 \% \mathrm{RH}$ \\
\hline Hot $(\mathrm{H})$ & $28.5{ }^{\circ} \mathrm{C}$ at $40 \% \mathrm{RH}$ & $37.5{ }^{\circ} \mathrm{C}$ at $33 \% \mathrm{RH}$ & $28.5{ }^{\circ} \mathrm{C}$ at $41 \% \mathrm{RH}$ \\
\hline Very Hot $(\mathrm{VH})$ & $28{ }^{\circ} \mathrm{C}$ at $37 \% \mathrm{RH}$ & $42.5{ }^{\circ} \mathrm{C}$ at $28 \% \mathrm{RH}$ & $28.1^{\circ} \mathrm{C}$ at $33 \% \mathrm{RH}$ \\
\hline Extremely Hot $(\mathrm{EH})$ & $28.1{ }^{\circ} \mathrm{C}$ at $43 \% \mathrm{RH}$ & $47.8{ }^{\circ} \mathrm{C}$ at $27 \% \mathrm{RH}$ & $28.3{ }^{\circ} \mathrm{C}$ at $44 \% \mathrm{RH}$ \\
\hline
\end{tabular}

Table 5. Shows the different exposure conditions from Stolwijk and Hardy ${ }^{13}$. 
Rectal Temperatures for Warm/Hot Exposures

a)

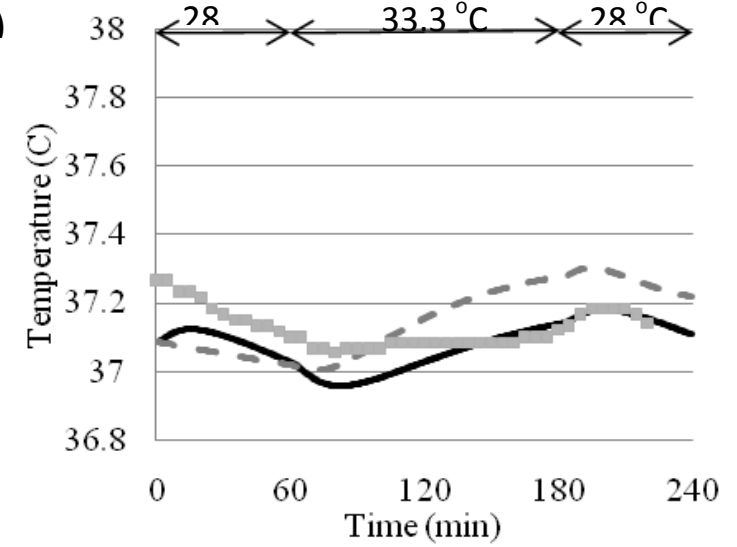

c)

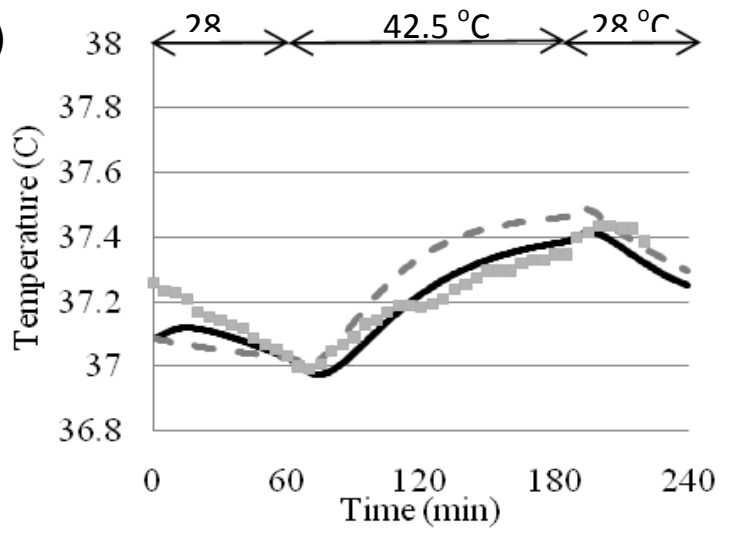

b)

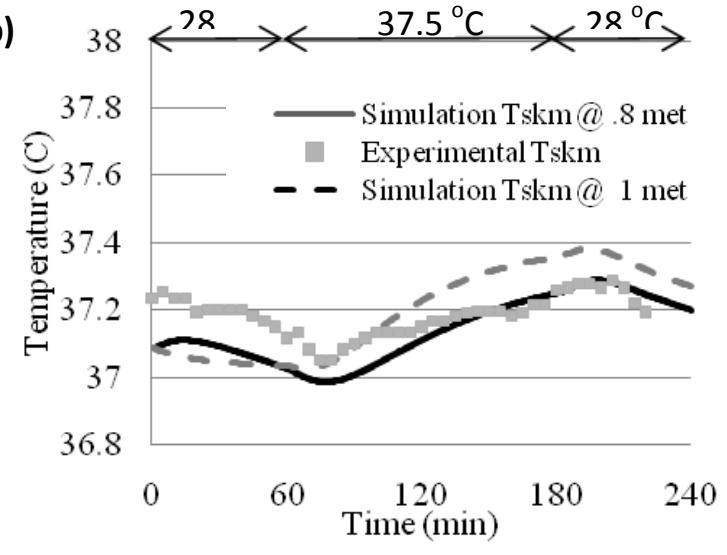

d)

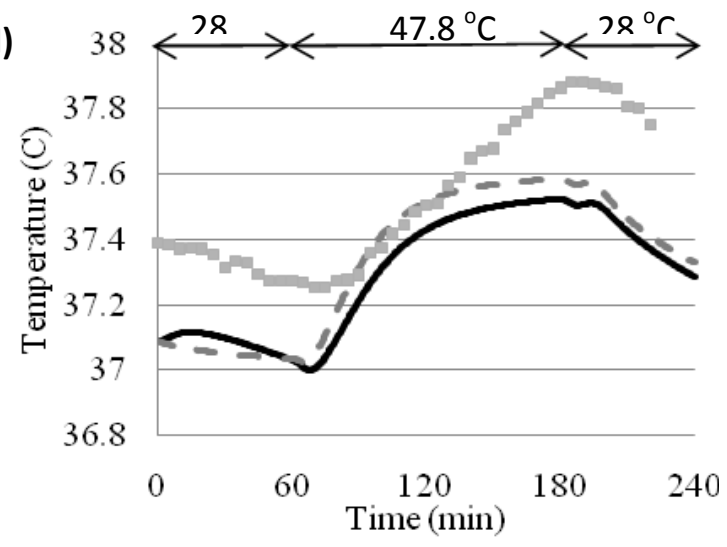

Figure 2. The simulated and experimental rectal temperature for the Stolwijk and Hardy ${ }^{13} \mathrm{~W}, \mathrm{H}$, $\mathrm{VH}$, and EH, environments at an air velocity of $.1 \mathrm{~m} / \mathrm{s}$. 
Mean Skin Temperatures for Warm/Hot Exposures
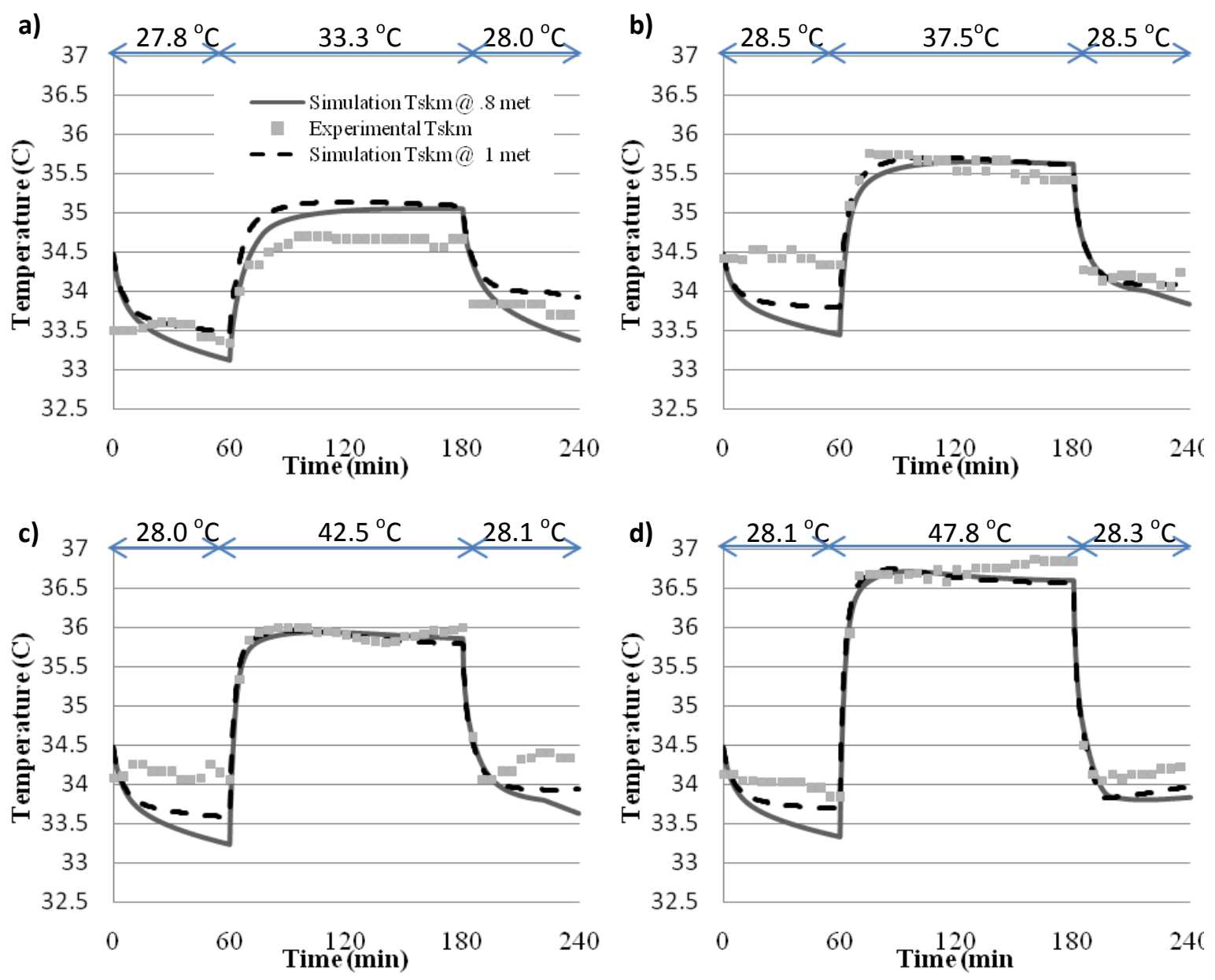

Figure 3. The simulated and experimental mean skin temperature for the Stolwijk and Hardy ${ }^{13}$ $\mathrm{W}, \mathrm{H}, \mathrm{VH}$, and $\mathrm{EH}$, environments at an air velocity of $.1 \mathrm{~m} / \mathrm{s}$.

\section{Cold Cases}

The cold environment response of the model was tested against a set of human subject experiments performed by Wagner and Horvath ${ }^{14}$. These experiments were performed on 10 men and 10 women between the ages of 20 and 30 and 10 men and 7 women between the ages of 51 and 72. The Fiala "standard man" physical model is the model used in the corresponding simulations and will be compared to the experimental results for the men of ages 20 to 30. Those subjects have a surface area of $1.89+/-.02$, a weight of $70.8+/-1.4 \mathrm{~kg}$, and a body fat percentage of $10.6+/-$ 9. In the experiment, the subjects were exposed to $10[\mathrm{C}]$ air for a period of $2 \mathrm{hrs}$. The simulation was run for a pre-exposure time of $60 \mathrm{~min}$ in a $25[\mathrm{C}]$ environment to allow for the time spent in the lab waiting for the experiment to begin. The air velocity near the subjects in the experiment was not recorded. A nominal air velocity of $.05 \mathrm{~m} / \mathrm{s}$ is used in the simulations. The measured activity level for the subjects in the 28 [C] environment was approximately $53\left[\mathrm{~W} / \mathrm{m}^{2}\right]$, which corresponds to about 0.9 met for the physical model simulated.

\section{Body Size Effects}

In order to investigate how the model responds for individuals of different size, the simulations were performed for a $5^{\text {th }}$ percentile man $(1.65[\mathrm{~m}]$ in height and $66.22[\mathrm{~kg}]$ in weight $)$ and a $95^{\text {th }}$ percentile man $(1.87[\mathrm{~m}]$ in height and $96.41[\mathrm{~kg}]$ in weight ${ }^{12}$ ). The size of each compartment was calculated as earlier described in section II-B. The percent body fat was calculated according to the method described by Montgomery ${ }^{11}$. The $5^{\text {th }}$ percentile percent 
body fat was calculated to be $14 \%$ body fat. The $95^{\text {th }}$ percentile body fat was calculated to be $18 \%$ body fat. The chosen environments to simulate were the $10[\mathrm{C}]$ cold environment and the $42.5[\mathrm{C}]$ warm environment from the earlier presented cases. The $50^{\text {th }}$ percentile man is shown next to the other results. It is the Fiala "average man" results.

Results for the Cold $10{ }^{\circ} \mathrm{C}$ Environment
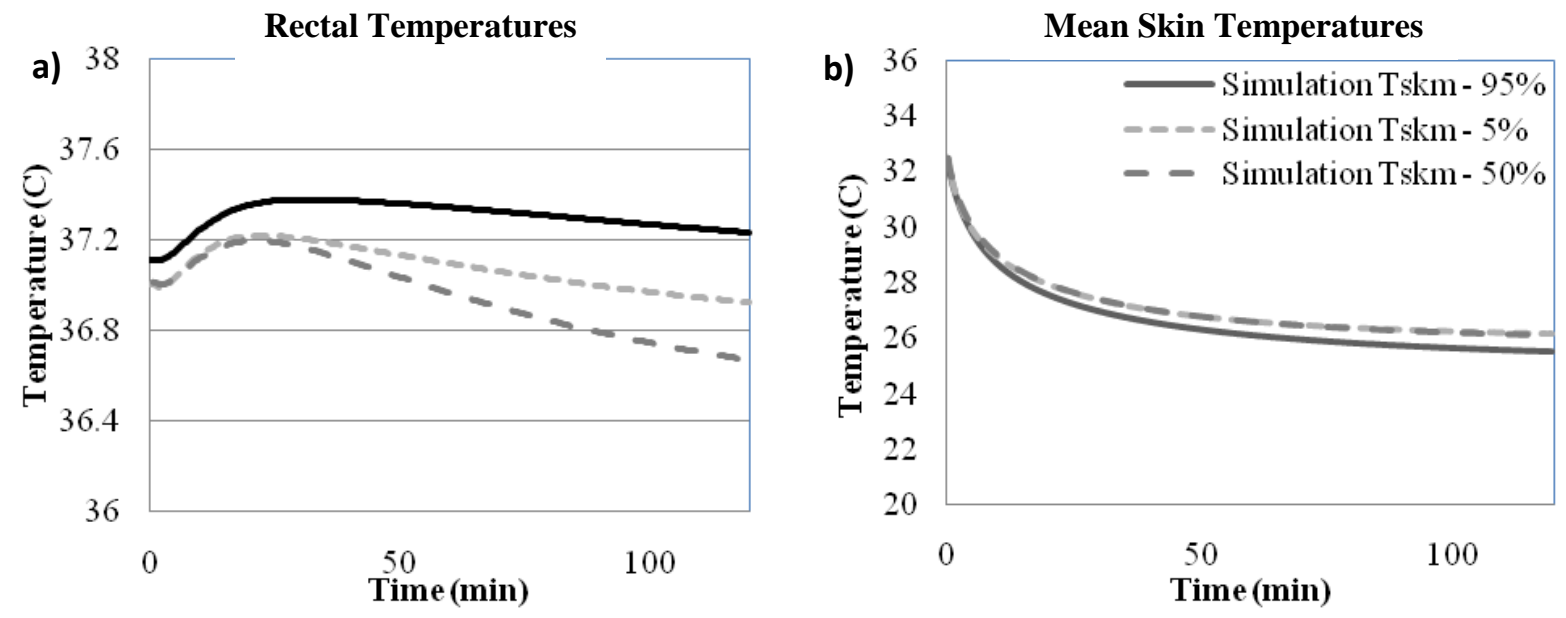

Figure 4. This figure shows the results for the simulated (a) rectal temperatures (b) mean skin temperatures of the $5 \%, 50 \%$, and $95 \%$ man for the cold Wagner and Horvath ${ }^{14}$ environment at an air velocity of $0.05 \mathrm{~m} / \mathrm{s}$, exposure wall and air temperatures of 10 [C] at $40 \% \mathrm{RH}$.

Varied Body Size Results for the 42.5 C Environment

Rectal Temperatures

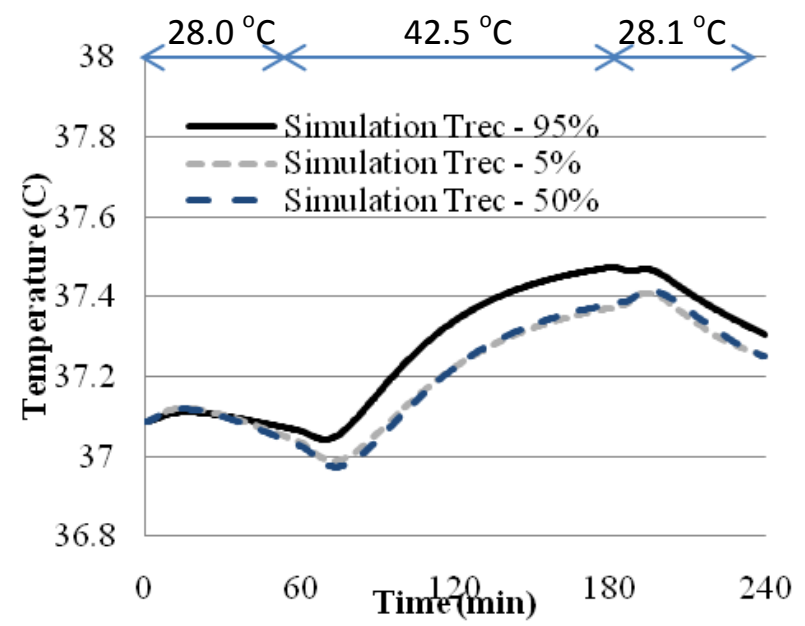

Mean Skin Temperatures

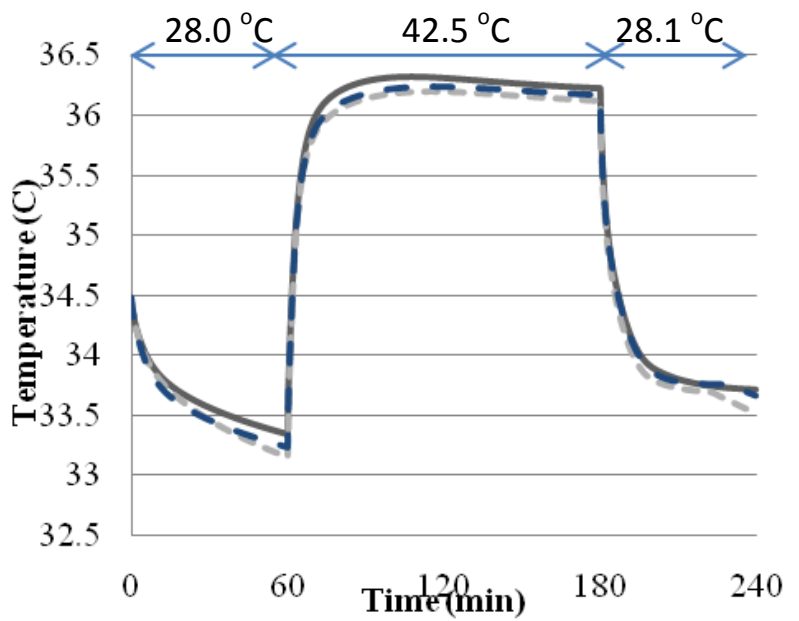

Figure 3. The simulated and experimental mean skin temperature for the $5 \%, 50 \%$, and $95 \%$ body sizes for the Stolwijk and Hardy ${ }^{13}$ VH environments at an air velocity of $.1 \mathrm{~m} / \mathrm{s}$.

\section{Discussion}

\section{A. Validation Criteria}

Validation of the model presumes some criterion for deciding whether or not the experimental and simulation results agree. Haslam and Parsons ${ }^{15}$ report a "maximum average standard deviation" of +/- 0.5 [C] for rectal temperatures in a survey of 80 different sets of experimental results for different environments and activity levels for 
a total of 590 individuals. They concluded that a model could produce reasonable results if it agreed within that range. Wissler ${ }^{5}$ presented the same $+/-0.5[\mathrm{C}]$ range of useful results. A difference of $+/-.5[\mathrm{C}]$ between the interior node, core, of the abdomen of the model and the measured rectal temperature will be used as the criterion for agreement in this discussion.

For mean skin temperatures, Haslam and Parsons ${ }^{15}$ reported that a model would agree with experimental results if the predicted and experimental results agreed within the "maximum average standard deviation" of $1.6[\mathrm{C}]$. Wissler ${ }^{5}$ stated that $1[\mathrm{C}]$ was a reasonable criterion for mean skin temperature agreement. However, the methods

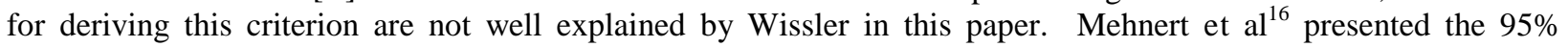
confidence level for 1399 experiments involving 377 subjects to be $+/-1.5$ [C]. This is close to the value reported by Haslam and Parsons ${ }^{15}$ of $1.6[\mathrm{C}]$. Therefore, $1.5[\mathrm{C}]$ will be the criteria used in this paper to determine agreement between experimental and simulated results of mean skin temperatures.

The mean skin temperature of the model used for comparison to experimental results is an area weighted mean skin temperature. All of the experimental results for mean skin temperature referenced in this thesis are measured by placing thermocouples on the skin to measure local skin temperatures. The method used to average the local temperatures is not always stated in the authors' reports and may vary with studies and authors.

\section{B. Core Temperatures}

\section{Thermoneutral Exposures}

Stolwijk and Hardy ${ }^{13}$ recorded rectal temperatures in a thermoneutral environment of 28 [C], 31\% RH, shorts, $0.1 \mathrm{~m} / \mathrm{s}$, for 3 subjects for 4 hours. The rectal temperatures tended to level out at approximately 37.05 [C]. Wagner and Horvath ${ }^{14}$, report a rectal temperature of approximately 36.8 [C] for a similar environment of 28 [C], $40 \%$ RH, shorts, $0.05 \mathrm{~m} / \mathrm{s}$ for 10 male subjects that are similar in physical characteristics to the "average man." The simulated steady state neutral condition mean skin temperature from Table 4 is 37.09 [C], which shows that model and experiments agree in this case according to the criteria of $+/-0.5$ [C] stated previously.

\section{Different Core Temperatures}

The model hypothalamus tracks $0.3+/-0.05$ [C] above the blood pool temperature and $0.1+/-0.05[\mathrm{C}]$ above the rectal temperature. In all simulations, the hypothalamus, rectal, and blood pool temperatures tended to maintain these approximate offsets independent of environment temperatures. This agrees with results presented by Nelson et al ${ }^{9}$ in a voxel model and Nunneley and Nelson ${ }^{17}$ in a numerical model of selective brain cooling ${ }^{18}$. The voxel model simulates hypothalamus temperatures 0.2-0.4 [C] above the blood pool temperature and the rectal temperature in between the hypothalamus and blood pool for the four Stolwijk and Hardy ${ }^{13}$ experiments. Nunneley and Nelson also show the "deep brain temperature" to be 0.2-0.3 [C] above the arterial temperature of blood supplied to the brain in 6 different simulations.

\section{Warm/Hot Exposure Rectal Temperatures}

In the 33.3 [C] environment, Figure 2-a, the largest difference between the experimental and simulated results occurs at the start of the pre-exposure time. This difference is not important because the thermal state of the subjects prior to this point is not reported, and the first 60 [min] is simply an equilibration period. During the 33.3 [C] room exposure, the difference between the predicted and experimental rectal temperature at 0.8 [met] agreed with experimental results with a maximum difference of 0.1 [C] occurring at about 80 [min]. At 1 [met], the maximum difference is 0.15 [C], which occurs at about 180 [min]. Both metabolic rates, 0.8 and 1 [met], agree with experimental results within the criterion of $+/-0.5$, but the 0.8 [met] exhibits closer overall agreement. The 0.8 [met] simulation tends to match the trends of the experimental data better than the 1 [met] simulation.

The 37.5 [C] simulation, Figure 2-b, shows results similar to those in the 33.3 [C], Figure 2-a, environment with the maximum difference between experimental and simulation occurring during the pre-exposure equilibration phase. During the actual hot exposure time, the maximum difference between the 0.8 [met] simulation and experimental results is approximately 0.1 [C], which occurs at about 90 [min] (30 [min] after the 28.5 [C] equilibrium period). At the $120 \mathrm{~min}$ mark, the 0.8 [met] simulation and experimental become virtually indistinguishable in value. The largest difference between the 1 [met] simulation and the experimental is 0.1 [C] also, but the difference occurs at the 180 [min] mark. The 1 [met] does not match the trends of the experimental results as well as the 0.8 [met] results. The 0.8 [met] rectal temperatures show a slight decline during the first 15 [min] of the 37.5 [C] room that is consistent with the experimental results. However, both the 0.8 [met] and 1 [met] simulations stay within the $+/-0.5$ [C] agreement criterion. This shows that the rectal temperatures are not sensitive to the low metabolic rates of the subjects in these experiments.

The 42.5 [C] environment, Figure 2-c, shows the best agreement between simulation and experimental temperatures of all the warm cases. The simulation and experimental values are a maximum of 0.05 [C] apart after 130 [min] of the exposure for the 0.8 [met] simulation and 0.15 [C] for the 1 [met] simulation. While both 
simulations agree within $+/-.5[\mathrm{C}]$, the 0.8 [met] matches the experimental values more closely than the 1 [met] simulation in this case. Comparison of simulation results for times, $t=0$ to $t=60$ [s], for Figures 4-2 through 4-5 shows little difference though the environments are slightly different. The experimental values vary much more than the simulation values, which is expected since the thermal state of the subjects prior to the experiment is not controlled or known.

The difference between the experimental and simulated rectal temperatures for the $47.8[\mathrm{C}]$ environment, Figure 2-d, is a maximum of about 0.4 [C] for the 0.8 [met] simulation and about 0.34 [C] for the 1 [met] simulation, which occurred at 180 [min]. This does agree within the specified criteria, but it is obvious there is a lot more difference between the experiment and simulation in this case than in the other cases. This could be due to the subjects having a mildly elevated core temperature at the start of the experiment. The experimental value at the end of the preexposure period was approximately between 0.15 and $0.26[\mathrm{C}]$ higher than the other cases at the end of their preexposure time. A similar experiment performed by Gagge et al. ${ }^{19}$ showed a rectal temperature at the start of the hot exposure, 60 [min], of $37.1[\mathrm{C}$ ] and a peak rectal temperature at the end of the hot exposure, 180 [min], of approximately $37.5[\mathrm{C}]$, which agrees better with the simulation. The trends also differ between the experimental data and simulation. The simulation is leveling out at the end of the exposure, which indicates that the model is achieving a steady state temperature. The experimental temperatures are still increasing linearly, which indicates that subjects are not near steady state.

The rectal temperatures for the 33.3, 37.5, 42.5, 47.8 [C] environments are plotted in Figure 2. The model predicted the rectal temperatures more closely to the experimental data at a simulation metabolic rate of 0.8 [met] than at 1 [met]. The difference between the rectal temperatures predicted for the two metabolic rates decreased at higher environmental temperatures, which illustrates how vasodilation and sweating become the predominant factors in controlling internal temperature. As the core temperatures and mean skin temperatures increase relative to their respective set points, the sweating and vasodilation increase and overwhelm the thermal effects of the slightly increased metabolic rate. Thus, the larger metabolic rate has a reduced effect on the rectal temperatures at higher environmental temperatures.

The model predicted an initial, transient decrease in rectal temperature after entering each of the warm/hot environments (Figure 2). It also predicted a transient increase in rectal temperature after leaving the hot environments and entering the cool environments in all cases except the 47.8 [C]. These rectal temperature responses are overshoot by the thermoregulatory system and are more exaggerated for the 0.8 [met] results than the 1 [met] results. The thermoregulatory overshoot upon entering each of the hot environments is due to the increase in skin blood flow, which would warm the skin and increase the control responses, vasodilation and sweating. The increase in the vasodilation and sweating is the reason for the rectal temperature decrease because vasodilated blood is cooled ${ }^{20}$. The increase of rectal temperature after leaving the hot environment is due to the decrease in skin blood flow and overall whole body heat transfer. In the 33.3 [C] environment, the model predicted a drop of about 0.06 [C]. This matched the measured experimental drop of .05 [C] well. The model predicted an increase of about 0.05 [C] after leaving the $33.3[\mathrm{C}]$ environment, Figure 2-a, which is the same as the experimental increase. The amount of overshoot tends to decrease as the temperature of the hot environment increases in both the experimental and simulated results. This is reasonable because the control response would need to faster for more extreme changes in environment.

\section{Cold Exposures}

In the $10[\mathrm{C}]$ cold exposures, Figure 4 , the model predicted the initial transient peak in rectal temperatures and generally followed the same curvature of the experimental results with a $0.1[\mathrm{C}]$ offset, which is within the criteria for agreement. This offset is due to the 0.17 [C] difference in initial temperatures at the start of the experiment and possibly differences between the neutral set points of the experimental subjects and the model. The thermal state of the subjects prior to the start of the experiments is not reported, so there is no exact way to model the starting conditions. The initial conditions at the start of the experiment were simulated by placing the subject in a 25 [C] room for an hour to decrease the starting rectal and mean skin temperatures.

\section{Individual Size Effects}

The individual rectal temperatures show no appreciable difference between the "average man" and $5^{\text {th }}$ percentile man in the $42.5[\mathrm{C}]$ environment. The $95^{\text {th }}$ percentile man had a higher rectal temperature and than the average man and the $5^{\text {th }}$ percentile man in the $42.5[\mathrm{C}]$ environment. The $95^{\text {th }}$ percentile man warmed during the equilibration period while the other two (average and $5^{\text {th }}$ percentile) cooled. This is likely related to the higher body fat percentage for the $95^{\text {th }}$ percentile man of $18 \%$ versus $14 \%$ for the other two size models. The higher body fat percentage reduces the conduction between the core and skin. The cold $10[\mathrm{C}]$ environment displayed the largest variation with individual sizes. The 5\% individual and average man showed transient increases to similar rectal temperatures of $37.2[\mathrm{C}]$ at a time, $\mathrm{t}$, of 20 [min]. The $5 \%$ individual then decreased gradually to approximately 
$36.95[\mathrm{C}]$, while the average man decreased gradually to approximately $36.75[\mathrm{C}]$. The $95 \%$ individual was predicted to have a warmer rectal temperature than both the 5\% individual and average man. The $95 \%$ individual showed a transient increase in rectal temperature to $37.4[\mathrm{C}]$, then decreased gradually to $37.2[\mathrm{C}]$.

\section{Skin Temperatures}

6. Thermoneutral Exposures

Stolwijk and Hardy ${ }^{13}$ recorded mean skin temperatures in a stated thermoneutral environment of 28 [C], $31 \%$ $\mathrm{RH}$, shorts, $0.1 \mathrm{~m} / \mathrm{s}$, for 3 adult, male subjects for 4 hours. The mean skin temperatures tended to level out at approximately $33.5[\mathrm{C}]$. Wagner and Horvath ${ }^{14}$, report a mean skin temperature of approximately 34 [C] for an environment of 28 [C], $40 \% \mathrm{RH}$, shorts, $.05 \mathrm{~m} / \mathrm{s}$ for 10 male subjects that are similar in physical characteristics to the "average man." Wagner and Robinson ${ }^{21}$ recorded the mean skin temperatures of a range of male subjects of ages 10-67 in conditions of 30 [C], 20\% RH, $0.05 \mathrm{~m} / \mathrm{s}$, shorts and socks for 60 [min]. The males of age 19-26 matched the physical characteristics of the "average man." The mean skin temperature was $34.6[\mathrm{C}]$ at the end of the thermoneutral exposure and seemed to be approaching steady state. The simulated steady state neutral condition mean skin temperature from Table 4 is 34.48 [C], which shows that model and experiments agree according to the criteria of $+/-1.5[\mathrm{C}]$ stated previously.

\section{Warm Environments}

The skin temperatures for the warm environments are plotted in Figure 3. There was not any substantial difference between the .8 and 1 [met] in the warm or hot room part of any of the simulations. The .8 [met] mean skin temperature was always approximately $.3[\mathrm{C}]$ lower than the 1 [met] at the end of the pre-exposure period due to higher vasoconstriction, and tended to show the same difference during the post exposure times as well.

There is a high variability between the experimentally measured mean skin temperatures during the pre-exposure period of the hot environments even though the environment is fairly consistent from one test to the next. For example, the pre-exposure to the 33.3 [C], Figure 3-a, experiment shows a mean skin temperature of approximately $33.5[\mathrm{C}]$, and the pre-exposure for the $37.5[\mathrm{C}]$ environment, Figure 3-b, shows a mean skin temperature of $34.5[\mathrm{C}]$. The mean skin temperature showed good agreement across all the hot environment temperatures; Figure 3; with a maximum difference of $0.4[\mathrm{C}]$ occurring during the $33.3[\mathrm{C}]$ exposure, which is well within the $+/-1.5[\mathrm{C}]$ requirement for agreement between the simulation and experimental values.

\section{Cold Exposures}

In the cold environment of $10[\mathrm{C}]$, Figure 4, after an initial transient change the model's predicted mean skin temperature was slightly offset from the experimental by approximately $0.6[\mathrm{C}]$. This offset could be due to slight differences in the clothing or air velocity because the exact values of these parameters are not reported. This offset is within the $1.5[\mathrm{C}]$ allowable tolerance, so the model agrees with the experimental results. The initial transient difference between the model and the experimental values is due to a difference in starting conditions. The thermal state of the subjects at the start of experiments is unknown, so there is no way to really simulate the beginning of the experiment.

The local skin temperatures in the $10[\mathrm{C}]$ environment agreed with the experimental results with the exception of the forehead. The simulation predicted a temperature of approximately $25[\mathrm{C}]$. The experimental results showed a forehead temperature of approximately $30.5[\mathrm{C}]$. The reason for this discrepancy is unknown. Other cold exposure experiments such as studies by Budd and Warhaft ${ }^{22}$ and Raven and Hovath ${ }^{23}$, which are studies in 5 [C] exposures show results of approximately $30[\mathrm{C}]$ for the forehead. The difference between simulated and experimental forehead temperatures is most likely due to a difference in skin blood flow to the head between the model and the subject. Raven and Horvath ${ }^{23}$ suggest that the head and chest is insensitive to cold environments because as the extremities reduce skin blood flow, blood and, therefore heat, is redistributed to the head and thoracic regions.

\section{Conclusion}

A numerical model meeting NASA's basic requirements, which is the primary objective of this thesis, has been developed. These requirements from Kuznetz ${ }^{1}$ are:

- The model must be written in a current, widely supported programming language.

- The physical model must reflect the general layout and composition of the human body and be capable of running on a personal computer.

- The model must have the capability of simulating a range of individuals based on anthropomorphic inputs.

- The model must be capable of simulating disparate environmental conditions. 
- The must be capable of simulating current and future space suit concepts.

The model is written in Mathworks MatLab, which is a modern language that is supported and used widely in the scientific and technical community. The language is similar to $\mathrm{C}++$ and is updated and maintained frequently. The model runs quickly, approximately 2 [min] for a 4 hour simulation, on a HP HDX 16 Notebook with 4 GB of RAM and an Intel Core Duo P7350 processor on a Microsoft Windows Vista 64 bit operating system. The model consists of 636 nodes and a $4 \mathrm{hr}$ simulation requires approximately 10 seconds of computer time at a 20 second time step, which is the largest time step permitted for stable, accurate results. Code written in MabLab is capable of being compiled, but the current model has not been compiled. The user inputs of the current model are performed by modifying variables in an ".m" file, which is a MatLab function file.

A space suit of any configuration can be modeled given heat and evaporative transfer coefficients for each local surface node. The size of the body segments are resized to simulate an individual with a certain height, weight, and body fat percentage under a range of activity levels up to 10 met $^{20}$ and environmental conditions.

The model consists of 19 body segments to represent the general shape and geometry of the human body. These body segments are separated into layers that represent the major tissue divisions of each segment. The body segments are separated into sectors to allow the simulation of different environmental conditions around the different sides of the body segments. Angular conduction from sector to sector is taken into account to improve the modeling of a person in such an asymmetric environment. One such environment is an astronaut with radiation shining on one side of him and the other side facing deep space.

The model was simulated for thermoneutral, warm, and cold environments for humans of several different anthropometric characteristics for comparison with experimental results. The experiments for an average man in a thermoneutral environment were performed by Stolwijk and Hardy ${ }^{13}$, Wagner and Horvath ${ }^{14}$, and Wagner et $\mathrm{al}^{21}$. Simulations were performed for an average man in hot environments of 33.3, 37.5, 42.5, and 47.8 [C] for comparison to experimental results from Stolwijk and Hardy ${ }^{13}$. In order to validate the results of the model in cold environments, temperatures for an average man in environments of 5, 10, and $20[\mathrm{C}]$ were simulated to compare the results of the model to results of experiments that were performed by Young et al. ${ }^{24}$ and Wagner and Horvath ${ }^{14}$. In addition to the average man, simulation results were generated for a 5th and 95th percentile man in environments of 42.5 and $10[\mathrm{C}]$. These results show that the size and composition of the person can make a difference in internal temperatures.

The model produced results that agreed well with the experimental results in all the comparisons performed. The simulated results were never further from the experimental results than the established criteria of a $+/-0.5$ [C] difference for the rectal temperatures and $+/-1.5[\mathrm{C}]$ difference for the mean skin temperatures. The only suspect result is the forehead skin temperature. However, there is not an exact criterion for comparing the simulated and experimental local skin temperatures anywhere on the body, so validity of the local temperatures cannot be stated for certain.

\section{Acknowledgments}

T. Miller thanks the Alabama Space Grant Consortium for providing the funding that made this research possible. Also, Dr. Gail Jefferson and Dr. James Downey of the University of South Alabama for their aid and input.

\section{References}

${ }^{1}$ Kuznetz, L. H. P. Technical Proposal for An Advanced Thermal Model of Humans in the Space Environment. 2001.

${ }^{2}$ Kuznetz, L. H. Control of Thermal Balance with a Liquid Circulating Garment Based on a Mathematical Representation of the Human Thermoregulatory System. PhD Dissertation 1975.

${ }^{3}$ Stolwijk, J. A. A Mathematical Model of Physiological Temperature Regulation in Man. Physiological and Behavioral Temperature Regulation 1970, 703-721.

${ }^{4}$ Kuznetz, L. H. A Two-Dimensional Transient Mathematical Model of Human Thermoregulation. American Journal of Physiology 1979.

${ }^{5}$ Wissler, E. H. Mathermatical Simulation of Human Thermal Behavior using Whole Body Models. In Heat Transfer in Medicine and Biology; Plenum Press: New York USA, 1985; Chapter 13, Vol. 1, pp 325-373.

${ }^{6}$ Mungcharoen, T.; Wissler, E. New Two-Dimensional Human Thermal Model. 1989, Vol. 85, 394-399.

${ }^{7}$ Fiala, D.; Lomax, K. J.; Stohrer, M. A Computer Model of Human Thermoregulation for a Wide Range of Environmental Conditions: The Passive System. Journal of Applied Physiology 1999. 
${ }^{8}$ Fiala, D.; Lomax, K. J.; Stohrer, M. Computer Prediction of Human Thermoregulation and Temperature Responses to a Wide Range of Environmental Conditions. Int J. Biometeorol 2001, 143-159.

${ }^{9}$ Nelson, D. A.; Charbonnel, S.; Curran, A. R.; Marttila, E. A.; Fiala, D.; Mason, P. A.; Ziriax, J. M. A HighResolution Voxel Model for Predicting Local Tissue. Journal of Biomechanical Engineering 2008.

${ }^{10}$ Werner, J.; Buse, M. Temperature profiles with respect to the inhomogeneity and geometry of the human body. Journal of Applied Physiology 1988, 65 (3), 1110-1118.

${ }^{11}$ Montgomery, L. D. A Model of Heat Transfer in Immersed Man. Journal of Biomedical Engineering, 1974, 1946.

${ }^{12}$ Winter, D. A. Biomechanics and Motor Control of Human Movement, 4th ed.; John Wiley and Sons, Inc.: Hoboken, NJ United States of America, Canada, 2009.

${ }^{13}$ Stolwijk, J. A.; Hardy, J. D. Partitional calorimetric studies of responses of man to thermal transients. Journal of Applied Physiology 1966, 21 (3), 967-977.

${ }^{14}$ Wagner, J. A.; Horvath, S. M. Influences of age and gender on human thermoregulatory responses to cold exposures. Journal of Applied Physiology 1985, 58 (1), 180-186.

${ }^{15}$ Haslam, R. A.; Parsons, K. C. Using computer based models for predicting human thermal responses to hot and cold environments. Ergonomics 1994, 37 (3), 399-416.

${ }^{16}$ Mehnert, P.; Malchaire, J.; Kampmann, B.; Piette, A.; Griefahn, B.; Gebhart, H. Prediction of average skin temperatures in warm and hot environments. European Journal of Applied Physiology 2000, 82, 52-60.

${ }^{17}$ Nunneley, S. A.; Nelson, D. A. Limitations on arteriovenous cooling of the blood supply to the human brain. European Journal of Applied Physiology 1994, 69, 474-479.

${ }^{18}$ Nelson, D. A.; Nunneley, S. A. Brain temperature and limits on transcranial cooling in humans: quantitative modeling results. European Journal of Applied Physiology 1998, 78, 353-359.

${ }^{19}$ Gagge, A. P.; Stolwijk, J. A.; Hardy, J. D. Comfort and Thermal Sensations and Associated Physiological Responses at Various Ambient Temperatures. In Environmental Research 1; Academic Press, Inc, 1967 ; pp 1-20.

${ }^{20}$ Fiala, D. Dynamische Simulation des menschlichen Warmehaushalts und der thermischen Behaglichkeit; Dissertation; De Montfort University Leicester and FH Stuttgart - Hochschule Fur Technik, 1998.

${ }^{21}$ Wagner, J. A.; Robinson, S.; Marino, R. P. Age and temperature regulation of humans in neutral and cold environments. Journal of Applied Physiology 1974, 37 (4), 562-569.

${ }^{22}$ Warhaft, N.; Budd, G. M. Body temperature, shivering, blood pressure, and heart rate during a standard cold stress in australia and antartica. Journal of Physiology 1966, 186, 216-232.

${ }^{23}$ Raven, P. R.; Horvath, S. M. Variability of physiological parameters of unacclimitized males during a two-hour cold stress. International Journal of Biometeorology 1970, 14 (3), 309-320.

${ }^{24}$ Young, A. J.; Muza, S. R.; Sawka, M. N.; Gonzalez, R. R.; Pandolf, K. B. Human thermoregulatory responses to cold air are altered by regular cold water immersion. Journal of Applied Physiology 1986, 60, 1542-1548.

${ }^{25}$ Raymond, G. G.; Roemer, R. B.; Horvath, S. M. A Mathematical Model of the Human Temperature Regulatory System - Transient Cold Exposure Results. IEEE Transactions on Biomedical Engineering 1976, BME-23 ( 6), 434-444.

${ }^{26}$ Pennes, H. H. Analysis of Tissue and Arterial Blood Temperatures in the Resting Human Forearm. J. Appl. Physiology 1948, 1, 93-122. 